\title{
Wrist circumference is a biomarker of adipose tissue dysfunction and cardiovascular risk in children with obesity
}

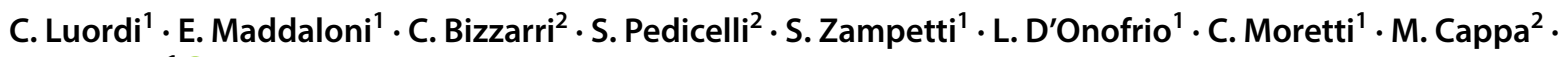 \\ R. Buzzetti ${ }^{1}$ (i)
}

Received: 17 May 2019 / Accepted: 14 July 2019

(C) Italian Society of Endocrinology (SIE) 2019

\begin{abstract}
Purpose To evaluate the relationship between wrist circumference, markers of adipose dysfunction, and cardiovascular risk in youths with obesity.

Methods In this cross-sectional study, we measured body mass composition by dual-energy X-ray absorptiometry, wrist circumference, waist-to-height ratio, fasting blood insulin, glucose, lipid profile, adiponectin, and leptin in 280 children with overweight/obesity and without diabetes (age: 7-18 years). Cardiovascular risk was estimated by "metabolic syndrome score" (MetS score).

Results Study participants had median [25th-75th percentile] wrist circumference of 17.5 [16.7-18.5] cm and waist-toheight ratio of 0.62 [0.59-0.67]. Lower adiponectin-leptin ratio was found among subjects in the upper 50th percentiles of wrist circumference $[0.17(0.09-0.36)$ vs. $0.38(0.16-0.79) ; p<0.001]$. Wrist circumference was independently associated with MetS score $(r=0.5 p<0.001)$. Among MetS score components, an independent association between wrist circumference HDLc, triglycerides, and systolic blood pressure was found $(r=-0.253 p<0.001 ; r=+0.204 p<0.001 ; r=+0.403$ $p=<0.001$, respectively). The coefficient of determination for MetS score was nominally higher when considering wrist circumference as independent variable ( $\left.\mathrm{Adj}-R^{2}=0.30\right)$ then when considering body mass index SD (Adj- $\left.R^{2}=0.28\right)$, waistto-height ratio (Adj- $R^{2}=0.26$ ) or truncal fat percentage (Adj- $R^{2}=0.01$ ). The addition of wrist circumference in age and gender adjusted models, accounting to any other anthropometric parameters, resulted in a significant improvement of the $\operatorname{Adj}-R^{2}(p<0.001$ for all).

Conclusions Our study shows that wrist circumference independently relates to adiponectin-leptin ratio and to the prediction of cardiovascular risk, suggesting it as an efficient and adjunctive anthropometric marker of cardiometabolic risk in children with obesity.
\end{abstract}

Keywords Childhood obesity $\cdot$ Wrist circumference $\cdot$ Adipokines $\cdot$ Cardiovascular risk $\cdot$ Insulin resistance

\section{Introduction}

In last decades, prevalence of overweight and obesity in childhood has substantially increased worldwide, with children becoming obese progressively early $[1,2]$. It is well established that childhood obesity is accompanied by a state of systemic metaflammation, hyperglycemia,

R. Buzzetti

raffaella.buzzetti@uniroma1.it

1 Department of Experimental Medicine, Sapienza University, Viale Regina Elena, 324, 00161 Rome, Italy

2 Unit of Endocrinology, Bambino Gesù Children's Hospital, Rome, Italy hyperinsulinemia, and insulin resistance (IR) [3, 4]. This results in an increased prevalence also of major cardiovascular risk factors, such as hypertension, dyslipidemia, and type 2 diabetes (T2D) [5, 6]. The identification of children with obesity who are more likely to develop these cardiometabolic conditions is still a challenge and we are not yet able to provide a targeted approach for cardiovascular prevention in this population. Over the years, various anthropometric measures of obesity have been identified [body mass index (BMI), waist circumference, waist-to-height ratio] and associated with cardiometabolic risk factors [7-9]. Recently, wrist circumference has emerged as a new sensitive anthropometric index of cardiometabolic risk in youths [10]. More specifically, we have previously shown 
that the bone component of wrist is associated with insulin resistance. This may raise the question of whether wrist circumference is independent from adiposity and associated with adipose tissue dysfunction. Moreover, it is not yet established whether wrist circumference during childhood correlates directly with the risk of developing cardiovascular disease later in adulthood.

In this study, we aim to evaluate the relationship between wrist circumference, markers of adipose tissue dysfunction, and a prediction score of cardiovascular risk in children and adolescents with obesity.

\section{Methods}

\section{Study design and population}

In this cross-sectional study, 280 children with overweight/ obesity aged $13 \pm 2$ years and attending the outpatient clinic of Bambino Gesù Children's Hospital in Rome, Italy, were consecutively enrolled from January 2015 to May 2017. Exclusion criteria were: diagnosis of diabetes mellitus, history of cardiovascular disease, bone disorders or neoplastic disease, previous or ongoing therapy with glucocorticoids, bisphosphonates, strontium ranelate, thiazolidinediones, and other bone-active agents.

\section{Anthropometric measurements}

All subjects enrolled in the study had a complete physical examination: weight $(\mathrm{kg})$, height $(\mathrm{cm})$, Body Mass Index (BMI) $\left(\mathrm{kg} / \mathrm{m}^{2}\right)$, standardized BMI (BMI SDS), waist circumference $(\mathrm{cm})$, wrist circumference $(\mathrm{cm})$, hip circumference $(\mathrm{cm})$, blood pressure $(\mathrm{mmHg}$ ), etc., (as subsequently reported in detail; Table 1).

Body weight, expressed in $0.1 \mathrm{~kg}$ intervals, was measured at fasting state in the morning. Harpendenstadiometers were used for body height, and every child was measured three times to the nearest millimeter according to the technique described by Cameron et al. [11].

In brief, the subject stood straight, with feet placed together and flat on the ground, heels, buttocks, and scapulae against the vertical backboard, and arms loose and relaxed with the palms facing medially. His head was carefully positioned in the Frankfurt plane, with the lower margins of the orbit in the same horizontal plane as the upper margin of the external auditory meatus.

BMI was calculated as body weight divided by heights squared $\left(\mathrm{kg} / \mathrm{m}^{2}\right)$ [12].
Table 1 Clinical and biochemical characteristics of the population

\begin{tabular}{ll}
\hline Variables & Total population \\
& $N=280$ \\
\hline Gender (\% of males) & $42.8 \%$ \\
Age (years) & $12.97 \pm 2.02$ \\
BMI $\left(\mathrm{kg} / \mathrm{m}^{2}\right)$ & $29.73(26.97-32.72)$ \\
BMI SD Italy & $2.14(1.74-2.46)$ \\
Waist circumference $(\mathrm{cm})$ & $99(91-107)$ \\
Wrist circumference $(\mathrm{cm})$ & $17.5(16.7-18.5)$ \\
Hip circumference $(\mathrm{cm})$ & $104.5(96.5-113.5)$ \\
Waist-to-height ratio $(\mathrm{cm})$ & $0.62(0.59-0.67)$ \\
Fat mass $(\%)$ & $40.5(37.9-43.5)$ \\
Lean mass $(\%)$ & $57(54.3-59.5)$ \\
Truncal fat $(\%)$ & $40.5 \pm 5.2$ \\
Systolic blood pressure $(\mathrm{mmHg})$ & $120.38 \pm 10.08$ \\
Diastolic blood pressure $(\mathrm{mmHg})$ & $65(60-73)$ \\
Serum creatinine $(\mathrm{mg} / \mathrm{dL})$ & $0.60(0.53-0.68)$ \\
Total cholesterol $(\mathrm{mg} / \mathrm{dL})$ & $157(142-175)$ \\
HDL cholesterol $(\mathrm{mg} / \mathrm{dL})$ & $46(40-52)$ \\
LDL cholesterol (mg/dL) & $94.5(78.6-108.0)$ \\
Triglycerides $(\mathrm{mg} / \mathrm{dL})$ & $79(60-108)$ \\
Gamma-glutamyltransferase $(\gamma-\mathrm{GT})(\mathrm{U} / \mathrm{L})$ & $14(12-19)$ \\
Uric Acid $(\mathrm{mg} / \mathrm{dL})$ & $5.07 \pm 1.01$ \\
25-OH Vitamin D (ng/mL) & $23.65(17.45-30.45)$ \\
Alkaline phosphatase $(\mathrm{ALP})(\mathrm{U} / \mathrm{L})$ & $464.0(276.5-607.5)$ \\
HbA1c (mmol/mol) & $34(33-36)$ \\
Fasting insulin $(\mathrm{IU} / \mathrm{ml})$ & $20.3(15.5-30.2)$ \\
Fasting C-peptide $(\mathrm{ng} / \mathrm{mL})$ & $1.93(1.51-2.45)$ \\
HOMA-IR index & $4.37(3.24-6.40)$ \\
MATSUDA index & $2.03(1.49-2.99)$ \\
TSH (mU/L) & $2.46(1.79-2.22)$ \\
C-reactive protein $(\mathrm{mg} / \mathrm{L})$ & $0.24(0.09-0.52)$ \\
Adiponectin $(\mu \mathrm{g} / \mathrm{dL})$ & $6.80[3.95-10.50]$ \\
Leptin (ng/dL) & $29.15[13.14-51.38]$ \\
Adiponectin/leptin ratio & $0.23[0.11-0.55]$ \\
\hline
\end{tabular}

The BMI of each individual was converted by smooth age-specific curves to a standard deviation score (BMI SDS) for the child's age as shown by Cole et al. [13].

Circumferences were measured using a metric nonstretching tape. Waist circumference was measured midway between the inferior margin of the lowest rib and the iliac crest in the horizontal plane at the end of normal expiration and recorded at the nearest $1.0 \mathrm{~cm}$; hip circumference was measured at the widest point over the buttocks; [14] waist-to-height ratio was obtained by dividing the waist circumference by height [15]. Dominant wrist circumference was measured with subjects in a seated position using a tension-gated tape measure positioned over the Lister tubercle of the distal radius and over the distal ulna $[16,17]$. 
The Lister tubercle, a dorsal tubercle of the radius, can be easily palpated [18] at the dorsal aspect of the radius around the level of the ulna head [19], about $1 \mathrm{~cm}$ proximal to the radiocarpal joint space [20].

Systolic and diastolic blood pressures were measured manually by calibrated sphygmomanometers.

\section{Clinical and biochemical parameters}

Blood samples were taken from each subject, after an overnight fast, for biochemical measurements with standard laboratory procedures.

An oral glucose tolerance test (OGTT) was performed for all children with overweight/obesity with the administration of glucose at $1.75 \mathrm{~g}$ per $\mathrm{kg}$ of body weight (maximum dose $75 \mathrm{~g}$ ); blood samples were obtained at baseline and every $30 \mathrm{~min}$, thereafter for $120 \mathrm{~min}$ for determination of serum glucose and insulin. Prediabetes was defined according to World Health Organization criteria (fasting blood glucose concentration between 110 and $126 \mathrm{mg} / \mathrm{dl}$ and/or $2 \mathrm{~h}$ post-glucose load between 140 and $200 \mathrm{mg} / \mathrm{dL}$ ). Estimates of insulin resistance were calculated using the homeostasis model assessment of insulin resistance (HOMA-IR), defined by fasting insulin and fasting glucose [21]. The Matsuda index was calculated with the formula: $(10,000 /$ square root of [fasting glucose $\times$ fasting insulin] $\times$ [mean glucose $\times$ mean insulin during OGTT]) [22]. The quantification of serum concentrations of adiponectin and leptin levels was determined using quantitative enzyme-linked immunosorbent assay (ELISA) (R\&D SYSTEMS).

\section{Dual-energy X-ray absorptiometry (DXA)}

Body composition was measured by DXA (QDR-4500A, Hologic, Inc.; Bedford Massachusetts).

Fat mass and lean mass were expressed as percentage of total body weight. Truncal fat was also evaluated and expressed as percentage of truncal weight.

\section{Cardiovascular risk assessment}

Cardiovascular risk was estimated by the "metabolic syndrome score" (MetS), which has been previously related to cardiometabolic risk in children and validated for the prediction of cardiometabolic outcomes in adults. Briefly, Viitasalo et al. showed that MetS is a valid tool for research evaluating cardiometabolic risk in different age groups, including children, able to predict cardiovascular outcomes and mortality. MetS formula [waist circumference $(\mathrm{cm})+$ $\operatorname{insulin}\left(\frac{\mathrm{IU}}{\mathrm{ml}}\right)+$ blood glucose $\left(\frac{\mathrm{mg}}{\mathrm{dl}}\right)+\frac{\text { Triglicerides }}{\mathrm{HDL}}+$ systolic blood pressure $(\mathrm{mmHg})+$ diastolic blood pressure $(\mathrm{mmHg})$ was used as previously reported [23] and $Z$ scores were calculated within the enrolled population.

\section{Statistical analysis}

Values are expressed as the mean \pm standard deviation (SD) or as the median [25th-75th percentiles] for parametric and non-parametric continuous variables, respectively; categorical variables are expressed as proportion. The normal distribution was tested with the Shapiro-Wilk test.

Continuous variables were compared between two groups using the Student's $t$ test or the Kruskal-Wallis test when appropriate. The Chi-square test and the Fisher exact test were used to compare the distribution of categorical variables between groups. Spearman test was used to evaluate correlations between continuous variables. The associations found were then tested for potential confounding factors in multivariate analysis using general linear regression (GLM) models. Nested models were compared with likelihood ratio tests. Non-parametric variables were transformed into natural logarithm before being tested in the model.

A value of $p<0.05$ was considered as statistically significant with a statistical power of $80 \%$.

Statistical analysis was performed using STATA statistical software (v 12.1) and GraphPad Prism 8.0 was used for graphical representations.

\section{Results}

\section{Population features}

Among the 280 recruited children with overweight/obesity, 120 were boys and 160 were girls, with mean \pm SD age of $13 \pm 2$ years, a median [25th-75th percentile] BMI of 29.7 [27-32.7] kg/m² and a median BMI-SDS of 2.14 [1.74-2.46]. Median wrist circumference was 17.5 [16.7-18.5] cm, waist circumference was 99 [91-107] $\mathrm{cm}$, and waist-to-height ratio was 0.62 [0.59-0.67]. Systolic blood pressure was $120.4 \pm 10.1 \mathrm{mmHg}$, total cholesterol was 147 [142-175] mg/dl, HDL was 46 [40-52] (mg/dl), LDL was 94.5 [78.7-108] mg/dl, triglycerides were 79 [70-107] mg/dl, fasting insulin was 20.3 [15.5-30.2] IU/ $\mathrm{mL}$, C-peptide was 1.93 [1.51-2.45] ng/mL, HOMA-IR was 4.4 [3.2-6.4], and Matsuda index was 2.03 [1.49-2.99] (Table 1).

OGTT identified $47(16.8 \%)$ subjects with a condition of prediabetes. Subjects with prediabetes had higher BMI [30.87 (28.32-4.86) vs. $29.30(26.71-32.57) \mathrm{kg} /$ $\left.\mathrm{m}^{2} ; p=0.015\right]$, waist-to-height ratio [0.65 (0.61-0.68) vs. $0.62(0.59-0.66) ; p=0.012]$, systolic blood pressure $[123.26 \pm 12.11$ vs. $119.82 \pm 9.57 \mathrm{mmHg} ; p=0.043]$, 
Table 2 Correlations of anthropometric indexes with MetS score and adiponectinleptin ratio

\begin{tabular}{lccc}
\hline Correlation & $r$ & $p$ & $p^{*}$ \\
\hline Wrist circumference vs. MetS score & $\mathbf{0 . 5}$ & $<\mathbf{0 . 0 0 1}$ & $<\mathbf{0 . 0 0 1}$ \\
Truncal fat \% vs. MetS score & 0.03 & 0.62 & 0.030 \\
Total fat \% vs. MetS score & 0.08 & 0.38 & 0.40 \\
Waist-to-height ratio vs. MetS score & $\mathbf{- 0 . 6}$ & $<\mathbf{0 . 0 0 1}$ & $<\mathbf{0 . 0 0 1}$ \\
Wrist circumference vs. adiponectin-leptin ratio & $\mathbf{- 0 . 3 7}$ & $<\mathbf{0 . 0 0 1}$ & $<\mathbf{0 . 0 0 1}$ \\
Truncal fat \% vs. adiponectin-leptin ratio & $\mathbf{- 0 . 2 4}$ & $<\mathbf{0 . 0 0 1}$ & $<\mathbf{0 . 0 0 1}$ \\
Total fat \% vs. adiponectin-leptin ratio & $\mathbf{- 0 . 2 5}$ & $\mathbf{0 . 0 0 2}$ & $\mathbf{0 . 0 0 1}$ \\
Waist-to-height ratio vs. adiponectin-leptin ratio & $\mathbf{- 0 . 2 7}$ & $<\mathbf{0 . 0 0 1}$ & $<\mathbf{0 . 0 0 1}$ \\
\hline
\end{tabular}

Bold values represent significant associations fasting triglycerides [93.5 (68-131) vs. 76 (60-102) mg/ $\mathrm{dL} ; p=0.004]$, serum insulin [23.12 (18.93-39.24) vs. 19.82 (15.12-29.13) IU/ml; $p=0.025]$, C-peptide levels [2.22 (1.98-2.76) vs. 1.89 (1.42-2.37) ng/mL; $p<0.001]$, HOMAIR [5.1 (3.75-9.08) vs. 4.18 (3.12-6.07); $p=0.008]$, and lower Matsuda index [1.73 (0.76-2.22) vs. 2.1 (1.57-3.15); $p=0.020]$. Higher wrist circumference was not associated with a higher risk of prediabetes after correction for age and gender (odds ratio for 1 SD increase: 0.98 [0.69-1.40]; $p=0.923)$.

\section{Wrist circumference and markers of adipose tissue dysfunction}

In the whole population, median serum adiponectin level was $6.80[3.95-10.50] \mu \mathrm{g} / \mathrm{dl}$, serum leptin was 29.15 [13.14-51.38] ng/dl, and adiponectin-leptin ratio was 0.23 [0.11-0.55]. Adiponectin-leptin ratio was lower in subjects with prediabetes than subjects with normal glucose tolerance [0.11 (0.08-0.28) vs. $0.30(0.13-0.64) ; p<0.001]$ and was inversely associated with C-peptide $(r=-0.512 p<0.001)$, independently from confounders. Lower values of adiponectin-leptin ratio were found in subjects in the upper 50th percentiles of truncal fat percentage $[0.18(0.08-0.38)$ vs. 0.36 $(0.15-0.65)]$, independently from age and gender, in subjects in the upper 50th percentiles of wrist circumference [0.17 (0.09-0.36) vs. $0.38(0.16-0.79) ; p<0.001]$ and in those in the upper 50th percentiles of waist-to-height ratio [0.18 $(0.09-0.37)$ vs. $0.39(0.16-0.79 ; p<0.001]$. Both wrist and waist-to-height ratio remained significantly associated with adiponectin-leptin ratio (Table 2) also in the multivariate model accounting for all wrist circumference, waist-toheight ratio, gender, and age together.

Wrist circumference was directly associated with HOMAIR $(r=+0.277 p<0.001)$ independently from age, gender, and waist-to-height ratio $(p=0.012)$. However, this association was not confirmed when estimating insulin resistance with the OGTT derivate Matsuda index $(r=-0.143$ $p=0.13$ ).

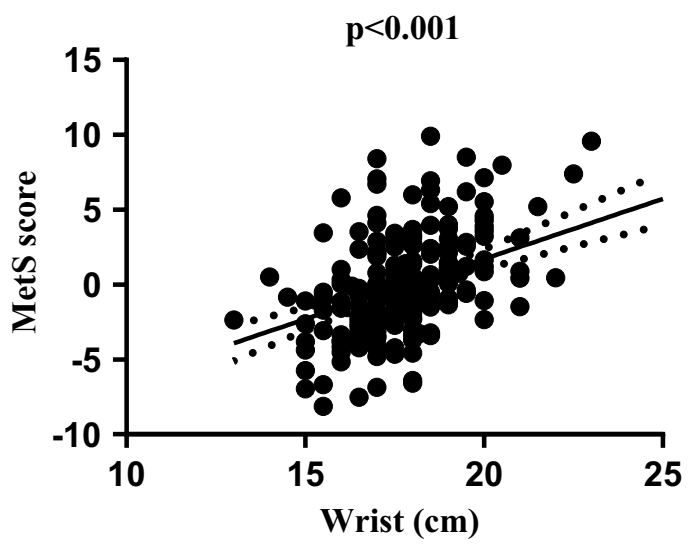

Fig. 1 Correlation between wrist circumference and MetS score $(r=0.5)$

Table 3 Adjusted coefficient of determination (Adj- $R^{2}$ ) representing the proportion of the variance for MetS score explained by the independent variables

\begin{tabular}{llll}
\hline Independent variable & Model 1 Adj- $R^{2}$ & Model 2 Adj- $R^{2}$ & $p$ value* \\
\hline Wrist circumference & 0.30 & N/A & N/A \\
BMI SD Italy & 0.28 & 0.36 & $<\mathbf{0 . 0 0 1}$ \\
Waist-to-height ratio & 0.26 & 0.37 & $<\mathbf{0 . 0 0 1}$ \\
Total fat (\%) & 0.07 & 0.22 & $<\mathbf{0 . 0 0 1}$ \\
Total truncal fat (\%) & 0.01 & 0.23 & $\mathbf{< 0 . 0 0 1}$ \\
\hline
\end{tabular}

Bold values represent significant $p$-values for difference between the two models

Model 1: independent variable + age + gender

Model 2: model 1+ wrist circumference

$N / A$ not applicable

${ }^{*} p$ value for difference between the two models

Moreover, we did not observe a correlation between truncal fat percentage and wrist circumference $(r=0.11 p=0.2)$.

\section{Wrist circumference and MetS score}

MetS score $(0.04 \pm 3.2$ in boys and $-0.21 \pm 3.3$ in girls $)$ was significantly and directly associated with wrist 
circumference, independently from gender and age $(r=0.5$ $p<0.001$ ) (Fig. 1).

The coefficient of determination [adjusted $R^{2}\left(\operatorname{Adj}-R^{2}\right)$ ] for MetS score was higher when considering wrist circumference as independent variable then when considering other anthropometric parameters with a well-recognized association with $\mathrm{CV}$ risk factors such as BMI SDS, waist-to-height ratio, total fat, and total truncal fat. Furthermore, the addition of wrist circumference in age and gender adjusted models accounting for one of the abovementioned anthropometric parameters always resulted in a significant improvement of the Adj- $R^{2}$ (Table 3).

Among the factors included in the MetS score formula, wrist circumference showed a significant association with HDLc, triglycerides, and SBP $(r=-0.253 p<0.001$; $r=+0.204 p<0.001 ; r=+0.403 p=<0.001$, respectively), independently from age, gender, and waist circumference ( $p=0.005 ; p=0.037 ; p=0.007$, respectively) or waist-toheight ratio ( $p<0.001 ; p=0.005 ; p<0.001$, respectively). An age- and gender-independent association between wrist circumference and fasting insulin concentration was found $(r=+0.272 p<0.001)$, which however disappeared after correction for waist circumference ( $p=0.22)$.Wrist circumference also showed a crude significant association with fasting blood glucose and diastolic blood pressure $(r=+0.159$ $p=0.011$ and $r=+0.157 p<0.001$, respectively), which were however dependent from age and gender $(p=0.11$ and $p=0.058$, respectively).

Truncal fat percentage and total fat percentage were not correlated with MetS $(r=0.03 p=0.62 ; r=0.08$; $p=0.38$, respectively). However, a significant association between MetS score and adiponectin-leptin ratio was found $(r=-0.468 p<0.001)$ (Table 2).

\section{Discussion}

The present study provides the first evidence that the measurement of wrist circumference is significantly related with serum adiponectin-leptin ratio and with an estimation of cardiovascular events' risk evaluated by MetS score in a population of children and adolescent with overweight/obesity.

These results are in accordance and expand previous data suggesting wrist circumference as a marker of cardiometabolic status in children.

\section{Wrist circumference and adipokines}

Excess fat is a major determinant of insulin resistance, associated with increased CV risk. Adipokines are key mediators of the association between childhood obesity and systemic low-grade inflammation [24]. Leptin is a major adipokine involved in the regulation of food intake and energy balance, linked to insulin resistance [25]. Adiponectin improves insulin sensitivity, regulates appetite, energy expenditure, and exerts numerous beneficial pleiotropic functions, including anti-inflammatory, antiproliferative, and anti-apoptotic effects. Hypoadiponectinemia has been found to be a strong indicator of both metabolic and vascular disorders [26]. The genetic susceptibility may play a major role: in previous studies, we observed that some polymorphisms of transcription factors as peroxisome proliferator-activated receptor-gamma2 Pro12Ala variant were associated with greater insulin sensitivity in childhood obesity [27] while others as adiponectin promoter region variants were significantly associated with lower insulin sensitivity, higher fasting glucose, and higher 2-h post-load glucose levels [28]. Overall, obesity and metabolic syndrome are distinguished by an increase in circulating leptin concentrations, in parallel to a drop in the levels of adiponectin. Consequently, the adiponectin-leptin ratio has been suggested as a marker of dysfunctional adipose tissue better correlating with insulin resistance than adiponectin or leptin alone [29].

We previously showed that wrist circumference is associated with insulin resistance in children with overweight and obesity [10], but no studies evaluated the association with adipokines. In that study, we clarified that the close relationship between wrist circumference and insulin resistance was explained by the skeletal frame size and not by the area of adipose tissue [10]. The present study confirms the independence of wrist circumference from the amount of body fat, as suggested by the absence of correlation with total and truncal fat percentage. However, the lower value of adiponectin-leptin ratio we found in subjects with larger wrist circumference suggests this anthropometric measure as an easy-to-detect marker of adipose tissue dysfunction. Of note, the correlation between adipokines and wrist circumference was stronger than the correlation with other validated cardiometabolic indexes, such as waist-to-height ratio or truncal fat. This might be explained by the fact that an increased amount of body fat is not always associated with an increase in adipose tissue dysfunction [30]. Therefore, anthropometric measures influenced by the amount of fat mass may not correctly represent adipose tissue dysfunction.

\section{Wrist circumference, insulin resistance, and cardiovascular risk (metabolic syndrome score)}

Both insulin resistance and adipose tissue dysfunction, which are effectively marked by wrist circumference, play a central role in the pathogenesis of cardiovascular disease. Before this study, we showed that wrist circumference is significantly and independently associated with cardiovascular risk factors such as hypertension and left ventricular structure, opening new perspectives in the prediction of cardiovascular diseases [31, 32]. Therefore, we also explored 
whether wrist circumference correlates with a validated marker of cardiovascular risk in children, the MetS score. MetS is recognized as an escalating major health risk in adults as well as in children and adolescents. Its prevalence ranges from 6 to 39\% depending on the applied definition criteria [33]. In children, the relatively low prevalence and the absence of a clear definition of the syndrome support the use of continuous MetS score as a marker of cardiovascular disease [34]. In particular, Viitasalo et al. showed that in middle-aged man, the risk of myocardial infarction and of cardiovascular death increased 1.38- and 1.56-fold, respectively, for a 1 SD increase in MetS score. Of note, they also showed that MetS score can be described as a single entity in all age groups, and therefore a valid tool for research evaluating cardiometabolic risk also in children, able to predict cardiovascular outcomes and mortality.

Our results showed that wrist circumference is associated with MetS score independently from age, gender, and other anthropometric indexes, suggesting that wrist circumference per se is a better predictor of MetS score than other anthropometric indexes. It provides additional information for the prediction of MetS score when considered on top of any other index tested in our study. The easy and simple way of measuring wrist circumference is a considerable advantage in clinical practice compared to serum biomarkers and even compared to BMI or waist circumference [10]. Wrist circumference measurement is not affected by clothing, respiration or by post-prandial state which can interfere with the determination of waist circumference [35].

\section{Strengths and limitations}

The strengths of the study include the large sample size and the description of adipose tissue to different levels: anthropometric, biochemical, and using DXA. This allowed a complete evaluation of children adiposity. This study also needs to be evaluated in light of some limitations. This is a cross-sectional study and for this reason cardiovascular risk was estimated only by cardiovascular risk score, in absence of longitudinal data. Moreover, we did not enroll normalweight children, therefore missing a control population to provide internal normative ranges.

\section{Conclusions}

In conclusion, our study shows that wrist circumference is related to adipose tissue dysfunction and to cardiovascular events' risk estimated by MetS score. Overall, this suggests that wrist circumference is an efficient anthropometric marker that should be routinely measured alone or on top of other indexes to improve the cardiovascular risk stratification in children with obesity.

Funding This study was funded by Grant of Italian Ministry of Health "Ricerca finalizzata" (RF-2010-2318911).

\section{Compliance with ethical standards}

Conflict of interest The authors declare that they have no conflict of interest.

Ethical approval The study was approved by the Ethical Committee of Bambino Gesù Children's Hospital in Rome.

Informed consent Written informed consent was obtained from the parents or guardians of the children included in this study, in accordance with the principles of the Declaration of Helsinki.

\section{References}

1. Weihrauch-Blüher S, Wiegand S (2018) Risk factors and implications of childhood obesity. Curr Obes Rep 7:254-259. https://doi. org/10.1007/s13679-018-0320-0

2. Steinbeck KS, Lister NB, Gow ML, Baur LA (2018) Treatment of adolescent obesity. Nat Rev Endocrinol 14:331-344. https://doi. org/10.1038/s41574-018-0002-8

3. Valerio G, Maffeis C, Saggese G et al (2018) Diagnosis, treatment and prevention of pediatric obesity: consensus position statement of the Italian Society for Pediatric Endocrinology and Diabetology and the Italian Society of Pediatrics. Ital J Pediatr 44:88. https:// doi.org/10.1186/s13052-018-0525-6

4. Ho HCH, Maddaloni E, Buzzetti R (2019) Risk factors and predicitve biomarkers of early cardiovascular disease in obese youth. Diabetes Metab Res Rev. https://doi.org/10.1002/dmrr.3134

5. Yanovski JA (2015) Pediatric obesity: an introduction. Appetite 93:3-12. https://doi.org/10.1016/j.appet.2015.03.028

6. Namazi N, Djalalinia S, Mahdavi-Gorabi A et al (2018) Association of wrist circumference with cardio-metabolic risk factors: a systematic review and meta-analysis. Eat Weight Disord. https:// doi.org/10.1007/s40519-018-0534-x

7. Maddaloni E, Cavallari I, De Pascalis M et al (2016) Relation of body circumferences to cardiometabolic disease in overweightobese subjects. Am J Cardiol. https://doi.org/10.1016/j.amjca rd.2016.06.044

8. Jayawardene WP, Lohrmann D, Dickinson S et al (2017) Clinical measures of obesity and cumulative cardiometabolic risk in adolescents. Clin Obes 7:11-21. https://doi.org/10.1111/cob.12171

9. de Quadros TMB, Gordia AP, Silva LR (2017) Anthropometry and clustered cardiometabolic risk factors in young people: a systematic review. Rev Paul Pediatr 35:340-350. https://doi. org/10.1590/1984-0462/;2017;35;3;00013

10. Capizzi M, Leto G, Petrone A et al (2011) Wrist circumference is a clinical marker of insulin resistance in overweight and obese children and adolescents. Circulation 123:1757-1762. https://doi. org/10.1161/CIRCULATIONAHA.110.012898

11. Cameron N (1978) The methods of auxological anthropometry. Hum Growth. Springer, Boston, pp 35-90

12. Freedman DS, Thornton JC, Pi-Sunyer FX et al (2012) The body adiposity index (hip circumference $\div$ height 1.5 ) is not a 
more accurate measure of adiposity than is bmi, waist circumference, or hip circumference. Obesity 20:2438-2444. https://doi. org/10.1038/oby.2012.81

13. Cole TJ, Bellizzi MC, Flegal KM, Dietz WH (2000) Establishing a standard definition for child overweight and obesity worldwide: international survey. BMJ 320:1240-1243. https://doi. org/10.1136/bmj.320.7244.1240

14. Bacopoulou F, Efthymiou V, Landis G et al (2015) Waist circumference, waist-to-hip ratio and waist-to-height ratio reference percentiles for abdominal obesity among Greek adolescents. BMC Pediatr 15:50. https://doi.org/10.1186/s12887-015-0366-z

15. Yoo E-G (2016) Waist-to-height ratio as a screening tool for obesity and cardiometabolic risk. Korean J Pediatr 59:425-431. https ://doi.org/10.3345/kjp.2016.59.11.425

16. Nyland J, Fried A, Maitra R et al (2006) Wrist circumference is related to patellar tendon thickness in healthy men and women. Clin Imaging 30:335-338. https://doi.org/10.1016/j.clini mag.2006.02.004

17. Campagna G, Zampetti S, Gallozzi A et al (2016) Excellent intra and inter-observer reproducibility of wrist circumference measurements in obese children and adolescents. PLoS One 11:e0156646. https://doi.org/10.1371/journal.pone.0156646

18. Hazani R, Engineer NJ, Cooney D, Wilhelmi BJ (2008) Anatomic landmarks for the first dorsal compartment. Eplasty 8:e53

19. Srinivas Reddy R, Compson J (2005) (i) Examination of the wrist - surface anatomy of the carpal bones. Curr Orthop 19:171179. https://doi.org/10.1016/j.cuor.2005.02.008

20. Strobel MJ (2002) Manual of arthroscopic surgery. Springer, Berlin Heidelberg

21. Matthews DR, Hosker JP, Rudenski AS et al (1985) Homeostasis model assessment: insulin resistance and beta-cell function from fasting plasma glucose and insulin concentrations in man. Diabetologia 28:412-419

22. Matsuda M, DeFronzo RA (1999) Insulin sensitivity indices obtained from oral glucose tolerance testing: comparison with the euglycemic insulin clamp. Diabetes Care 22:1462-1470. https ://doi.org/10.2337/diacare.22.9.1462

23. Viitasalo A, Lakka TA, Laaksonen DE et al (2014) Validation of metabolic syndrome score by confirmatory factor analysis in children and adults and prediction of cardiometabolic outcomes in adults. Diabetologia 57:940-949. https://doi.org/10.1007/s0012 5-014-3172-5

24. Bai Y, Sun Q (2015) Macrophage recruitment in obese adipose tissue. Obes Rev 16:127-136. https://doi.org/10.1111/obr.12242
25. Gruzdeva O, Borodkina D, Uchasova E et al (2019) Leptin resistance: underlying mechanisms and diagnosis. Diabetes Metab Syndr Obes 12:191-198. https://doi.org/10.2147/DMSO.S182406

26. Szewczyk-Golec K, Woźniak A, Reiter RJ (2015) Inter-relationships of the chronobiotic, melatonin, with leptin and adiponectin: implications for obesity. J Pineal Res 59:277-291. https://doi. org/10.1111/jpi.12257

27. Buzzetti R, Petrone A, Caiazzo AM et al (2005) PPAR-gamma2 Pro12Ala variant is associated with greater insulin sensitivity in childhood obesity. Pediatr Res 57:138-140. https://doi. org/10.1203/01.PDR.0000147728.62185.21

28. Petrone A, Zavarella S, Caiazzo A et al (2006) The promoter region of the adiponectin gene is a determinant in modulating insulin sensitivity in childhood obesity. Obesity (Silver Spring) 14:1498-1504. https://doi.org/10.1038/oby.2006.172

29. Frühbeck G, Catalán V, Rodríguez A et al (2019) Adiponectinleptin ratio is a functional biomarker of adipose tissue inflammation. Nutrients. https://doi.org/10.3390/nu11020454

30. Bray GA, Heisel WE, Afshin A et al (2018) The science of obesity management: an endocrine society scientific statement. Endocr Rev 39:79-132. https://doi.org/10.1210/er.2017-00253

31. Zampetti S, Campagna G, Lucantoni F et al (2018) Wrist circumference is associated with increased systolic blood pressure in children with overweight/obesity. Hypertens Res 41:193-197. https://doi.org/10.1038/s41440-017-0006-0

32. Zampetti S, Campagna G, Leto G et al (2018) Relation between wrist circumference and left ventricular structure in overweight children. Am J Cardiol 121:1624-1628. https://doi.org/10.1016/j. amjcard.2018.02.057

33. Bussler S, Penke M, Flemming G et al (2017) Novel insights in the metabolic syndrome in childhood and adolescence. Horm Res Paediatr 88:181-193. https://doi.org/10.1159/000479510

34. Eisenmann JC (2008) On the use of a continuous metabolic syndrome score in pediatric research. Cardiovasc Diabetol 7:17. https ://doi.org/10.1186/1475-2840-7-17

35. Bosy-Westphal A, Booke C-A, Blöcker T et al (2010) Measurement site for waist circumference affects its accuracy as an index of visceral and abdominal subcutaneous fat in a Caucasian population. J Nutr 140:954-961. https://doi.org/10.3945/jn.109.118737

Publisher's Note Springer Nature remains neutral with regard to jurisdictional claims in published maps and institutional affiliations. 